\title{
Jeanne d'Arc, dux, chef de guerre
}

Les points de vue des traités en faveur de la Pucelle

Françoise Michaud-Fréjaville

\section{(2) OpenEdition \\ Journals}

Édition électronique

URL : https://journals.openedition.org/crm/732

DOI : $10.4000 / \mathrm{crm} .732$

ISSN : 1955-2424

Éditeur

Honoré Champion

\section{Édition imprimée}

Date de publication : 1 juin 2005

Pagination : 189-197

ISSN : $1272-9752$

Référence électronique

Françoise Michaud-Fréjaville, "Jeanne d'Arc, dux, chef de guerre », Cahiers de recherches médiévales [En ligne], 12 spécial | 2005, mis en ligne le 28 juin 2008, consulté le 15 décembre 2022. URL : http:// journals.openedition.org/crm/732 ; DOI : https://doi.org/10.4000/crm.732 


\title{
rigM
}

\author{
$-16-$ \\ Jeanne d'Arc, dux, chef de guerre. \\ Les points de vue des traités en faveur de la Pucelle
}

\author{
Et devant elle vont fuyant \\ Les ennemis, ce nul n'y dure. \\ Elle fait ce, mains yeux voians, \\ Et d'eulx va France descombrant, \\ En recouvrant chasteaux et villes. \\ Jamais force ne fu si grant, \\ Soient ou à cens ou à miles! \\ Et de nos genz preux et habiles \\ Principale chevetaine. \\ Telle force n'ont Hector ni Achilles! \\ Mais tout ce fait Dieu qui la menne. \\ (Christine de Pizan, Ditié de Jeanne d'Arc)
}

Dès l'été de 1429, beaucoup de ses contemporains considérèrent Jeanne d'Arc comme un chef de guerre, une «chevetaine $»^{2}$. Charles VII et l'armée royale avaient une femme inspirée parmi les chefs militaires. Et c'était merveille. Évidemment, le Bourgeois de Paris ne l'entendait pas ainsi : à ses yeux la jeune fille passait, en ce même été, pour la Pucelle armée des Armagnacs, une «créature qui était en forme de femme [...] qu'on nommait la Pucelle, qui c'était, Dieu le sait $»^{3}$.

Notre propos n'est pas de discuter de la nature réelle de l'autorité que Jeanne avait sur les seigneurs et capitaines qui l'entouraient, pas plus que sur ses talents militaires, son génie de la tactique et de la stratégie. Une abondante littérature s'est attachée, en particulier, à mettre en avant une valeur militaire exceptionnelle de la Pucelle. Ces travaux sont assez souvent très marqués par leur contexte historique, c'est-à-dire les années qui suivirent les défaites de 1870 et de 1940 , et par les convictions politiques et confessionnelles de leurs auteurs ${ }^{4}$. La question soulève de

\footnotetext{
${ }^{1}$ Christine de Pizan, Ditié de Jeanne d'Arc, éd. Angus J. Kennedy et Kenneth Varty, Oxford, 1977, v. 277-288, p. 35.

${ }^{2}$ On peut apprécier que si tôt «chef» ait un féminin - cheftaine - quand aujourd'hui certains s'interrogent sur le bien fondé de la création d'un genre féminin des fonctions jusqu'à présent masculines.

${ }^{3}$ Journal d'un Bourgeois de Paris de 1405 à 1449, éd. Colette Beaune, Paris, Librairie Générale française, (Livre de poche, coll. Lettres gothiques), 1990, § 519 (7 septembre 1429), p. 265.

${ }^{4}$ Hospes (pseudonyme), Jeanne d'Arc grand capitaine, sa campagne sur la Loire, la marche entre Reims et Paris, Orléans, Houzé, 1909. Il s'agit d'une réponse à la Jeanne d'Arc d'Anatole France (1909). R. Olivier, "Jeanne d'Arc, chef de guerre », Revue de la cavalerie
}

Cahiers de Recherches Médiévales (XII -XV s.), 12spé, 2005 
toute façon des passions et provoque les spécialistes de l'histoire militaires. En contrepoint des dithyrambes, les détracteurs de l'action de Jeanne, qui parfois rejoignent curieusement les zélateurs de son seul aspect spirituel et pacifiste, la rejettent au rang d'un simple porte-bonheur ou en font le jouet innocent, inconscient, voire pervers, d'un infâme entourage royal'.

Nous ne nous engagerons pas dans cette voie, mais relisant les textes, nous nous attacherons à voir comment, de 1429 à 1456, dix-huit traités en faveur de la Pucelle ${ }^{7}$ ont abordé, exploité ou esquivé ce qui devait faire le cinquante-troisième de la première série des chefs d'accusation du procès de Rouen (mercredi 28 mars 1431) : «Contrairement aux commandements de Dieu et des saints, ladite Jeanne a assumé, avec orgueil et présomption, la domination sur les hommes; elle s'est constituée chef et capitaine d'armée (caput et ducem exercitus), s'élevant parfois au nombre de $\mathrm{XVI}^{\mathrm{m}}$ hommes où se trouvaient princes, barons et autres nobles, que tous elle a fait servir militairement sous elle comme principal capitaine $»^{8}$. La jeune fille avait rétorqué qu'elle n'avait été «chief de guerre» que pour chasser les Anglais". L'accusation stigmatisait les réponses que Jeanne avait faites le 27 février précédent à la fin d'un long interrogatoire sur ses voix, ses épées, l'étendard, les combats d'Orléans et de Jargeau. Le notaire avait enregistré : «À la question, interrogée quelle compagnie lui donna son roi quand il la mit en œuvre, répondit qu'il lui bailla $\mathrm{X}$ ou $\mathrm{XII}^{\mathrm{m}}$ hommes; et que d'abord elle alla à Orléans, etc. $»^{10}$. On peut noter au

blindée, t. 106 (1979), p. 43-68. S. T. Richey, «Les qualités de Jeanne d'Arc en tant que chef militaire ", Bulletin de l'Association des amis du centre Jeanne d'Arc, 23 (1999), p.73-88.

${ }^{5}$ Histoire militaire de la France, t. 1, Des origines à 1715, P. Contamine (dir.), Paris, 1992, p. 195 : «Au gré des circonstances imprévisibles, la fortune pouvait passer dans l'un ou l'autre camp. L'intervention décisive de Jeanne d'Arc se produisit dans ce contexte: elle redonna du cœur à des troupes qui étaient déjà à pied d'œuvre ».

${ }^{6}$ Une vue d'ensemble, qui aurait besoin d'une mise à jour: Y. Grandeau, Jeanne insultée. Procès en diffamation, Paris, 1973.

${ }^{7}$ Mémoires et consultations en faveur de Jeanne d'Arc, par les juges du procès de réhabilitation, éd. Pierre Lanery d'Arc, Paris, 1889. L'éditeur voulait constituer un « complément» au tome V de l'édition des Procès de Jules Quicherat en publiant quinze traités dans leur intégralité. Pierre Duparc a réédité huit des traités au t. II de son édition du Procès en nullité de la condamnation de Jeanne d'Arc, Paris, Société de l'histoire de France, 1979. La réédition et la traduction (partielle) des traités a été lancée par P. Contamine pour la Société de l'histoire de France, elle est en cours. On y trouvera en outre la note de Jean Dupuy dans son Breviarum historiale retrouvée par Léopold Delisle (cf. L. Delisle, Nouveau témoignage relatif à la mission de Jeanne d'Arc. Communication faite à l'Académie des Inscriptions et Belles-Lettres le 23 octobre 1885, Paris, 1885), texte utilisé dans la dernière partie de cet article.

${ }^{8}$ Traduction de P. Champion, Procès de condamnation de Jeanne d'Arc, Paris, 1921, t. I, p. 241 (texte latin), t. II, p. 182 (traduction). Dans l'édition de P. Tisset, Procès de condamnation Jeanne d'Arc, Paris, Société de l'histoire de France, 1969, t. I, p. 262.

${ }^{9}$ Ibid. : Quantum ad factum d'estre chief de guerre gallice ipsa alias de hoc respondit : et si ipsa fuit caput guerre, hoc fuit pro verberando Anglicos ("Quant au fait «d'estre chef de guerre » comme on dit en français, elle y répondit que si elle avait été chef de guerre, c'était pour renvoyer les Anglais »).

${ }^{10} I b i d$., t. I. p. 58-59 (texte latin), et t. II, p. 52 (traduction). 
passage, en un mois, l'accroissement de plus de la moitié du nombre des hommes ${ }^{11}$, le glissement de l'acte royal qui confiait à Jeanne une partie de l'armée à une usurpation de la Pucelle qui s'instituait elle-même comme capitaine (se constituendo caput et ducem exercitus), bafouait l'ordre humain naturel en se plaçant de son propre mouvement au dessus de ses supérieurs sociaux, les nobles (barones), et des techniciens spécialistes, les capitaines, et justifiait ainsi l'accusation de présomption.

L'analyse des traités, qui reste dans l'ensemble largement encore à faire ${ }^{12}$, permet de constater que la mise en question de la place tenue dans l'armée par Jeanne a été évitée avec une constance qui ne peut que révéler le malaise profond des gens d'Église en face d'une situation imprévue, inouïe et qui demeure, si l'on y songe, encore unique dans l'Histoire non légendaire.

Un premier groupe de textes, ceux de Jacques Gélu ${ }^{13}$, Jean Gerson ${ }^{14}$, Jean Dupuy $^{15}$, Henri de Gorkum ${ }^{16}$, doit être examiné à part et en tête parce qu'antérieur à la capture de Jeanne d'Arc. Ces oeuvres ne peuvent être les réponses à des accusations non encore formulées par un tribunal hostile, mais elles sont des opinions personnelles sur les questions que se posait le parti de Charles VII, et certainement le reflet des réactions d'une bonne part des élites armagnacques.

Gélu, répondant à une demande de consultation royale, après avoir exposé combien était à la fois juste mais menacée et fragile la situation du roi légitime Charles VII, rappelait, entre autres choses, que cette fillette adolescente affirmait venir de par Dieu et que «si on la laissait prendre la tête de l'armée», agir en $d u x$, " elle vaincrait» ces ennemis qui semaient la terreur ${ }^{17}$. Elle avait certes chassé les Anglais de leurs places sur la Loire et son succès pouvait être la preuve de la puissance de Dieu, encore que la prudence dût être de rigueur. Ce que demandait le roi à l'évêque d'Embrun n'était pas de trancher sur le choix de laisser une jeune fille mener ou non une armée, pour cela l'avis de Gélu serait venu trop tard, mais si la Pucelle, une fille en chair et en os, était susceptible d'avoir été envoyée par Dieu. Gélu classe ses réponses en quatre thèmes dont le troisième devrait toucher à notre propos : la sagesse divine peut-elle dévoluer (committere) au sexe féminin les choses qui sont de la compétence des hommes (quae viris competunt) ${ }^{18}$. On voit que l'évêque ne peut pas penser autrement qu'en cas général, et son discours se perd

${ }^{11}$ Les défaites anglaises sur la Loire, puis la campagne du sacre en devenaient en quelque sorte explicables, mais d'un autre côté cela aggravait le pouvoir envoûtant de Jeanne sur les hommes d'armes; tout demeure ambigu.

${ }^{12}$ J. M. Pinzino, «Speaking of angels : a fifteenth-century bishop in defense of Joan of Arc's mystical voices », dans Fresh Verdicts on Joan of Arc, éd. B. Wheeler et C. T. Wood, New York, 1996, p. 161-176. L'évêque en question est Élie de Bourdeilles.

${ }^{13}$ Mémoires..., p. 565-600 : Jacobi Gelu archiepiscopi dissertatio. Cf. O. Bouzv, « Le traité De adventu Johanne », Bulletin de l'Association des amis du Centre Jeanne d'Arc, 1992, p. 2940.

${ }^{14}$ Jean Gerson, Opusculum super facto puelle [De mirabili victoria], Procès en nullité..., éd. Duparc, t. II, p. 33-39.

${ }^{15} \mathrm{Cf}$. note 6. Voir A. Dondaine, «Le frère prêcheur Jean Dupuy, évêque de Cahors et son témoignage sur Jeanne d'Arc », Archivum fratrum praedicatorum, t. XII (1942), p. 167-184.

${ }^{16}$ Procès de Jeanne d'Arc, éd. Quicherat, t. II, p. 411-421.

${ }^{17}$ Mémoires..., p. 571-572: Ut si dux exercitus seu armis se immisceat (...), vinceat.

${ }^{18}$ Ibid., p. 579-580. 
dans des considérations sur les hommes punis pour n'avoir pas su garder leurs prérogatives, puis sur les prophéties féminines et les Sibylles, pour finir par constater que Dieu ne se trompe jamais, qu'Il peut imposer à son gré des mutations à l'ordre de Nature. Dans le cas précis, Il peut ordonner que « la Pucelle soit à la tête de soldats et même les commande" (quod Puella armatis viris praesset et eos regeret), qu'elle combatte et soit victorieuse de gens redoutables et expérimentés (fortissimos et exercitatissimos debellaret et vinceret). Cela est possible car ce qu'Il ordonne fait loi et la nature s'y plie. Mais si l'initiative ne vient pas de Lui, il y a de la part de celui qui transgresse la nature, péché de présomption. Le mot presumptio fut bel et bien écrit et les risques d'orgueil et d'outrecuidance ont été abordés par l'évêque d'Embrun, avant même d'avoir été assenés par le tribunal de Rouen.

Gerson, de son côté, considère que la guerre ayant pour but de rendre à un souverain légitime tout son royaume est une guerre juste qui appartient au projet divin $^{19}$. Dans ces conditions, et malgré les moqueries, les gens du roi ont cru à la parole de la jeune fille et se sont exposés aux risques de la guerre sous et avec elle (sub ea et cum ea exposuerunt [...] sub ea juvencula et muliercula militantes) et le résultat fut la victoire. Sans aucun doute pour Gerson, dans un premier temps de son exposé, Jeanne semble avoir été un chef de guerre auquel le conseil du roi et les militaires se sont confiés corps et âmes, c'est vraiment le cas de le dire. D'une décision personnelle royale, il ne dit rien. Si les événements ensuite ne tournent pas bien, le souverain sera innocent des errements commis. Dans sa péroraison, le prudent théologien insiste sur le caractère circonstanciel et donc unique de la transgression des rôles homme-femme dans les vêtements et la coupe des cheveux. Surtout il revient sur la présence à l'armée de Jeanne avec une expression fort intéressante : Jeanne a été choisie par Dieu comme «une porte-étendard» (vexilliferam). On voit ainsi que Gerson connaît parfaitement les circonstances dans lesquelles la Pucelle a saisi en personne son étendard au combat, devenant ainsi incapable de porter les coups, comme elle-même l'expliqua lors du procès. Mais par ce fait il la rangeait désormais non plus au nombre des chefs, mais plutôt parmi les auxiliaires. Même si on ne peut le compter au nombre de ceux qui virent en la Pucelle une simple mascotte, cum ea restait toujours plus acceptable que sub ea pour l'élite dirigeante.

Henri de Gorkum ${ }^{20}$, dans la très courte évocation de la personne de Jeanne par laquelle commence son traité, la présente également avec le vocabulaire des chefs militaires, mais précédé d'un quasi qui laisse à penser que pour lui il s'agit d'une comparaison individuelle plutôt que de l'exercice d'une fonction: viget industria quasi peritus dux exercitus ad antificiosam exercitus institutionem ${ }^{21}$.

Enfin Jean Dupuy, dans ce qui n'est pas exactement un traité, mais une réflexion incise dans son Breviarum historiale, annonce d'emblée qu'il "préfère pas-

${ }^{19} \mathrm{P}$. Contamine, « La théologie de la guerre à la fin du Moyen Âge : la guerre de Cent Ans futelle une guerre juste?", Jeanne d'Arc, une époque, un rayonnement. Colloque d'histoire médiévale, Orléans, octobre 1979, Paris, 1982, p. 9-21.

${ }^{20}$ Heinrich von Gorkum (sic pour Hendrik van Gorkum), De quadam puella, Procès de Jeanne $d^{\prime} A r c$, éd. Quicherat, t. V, p. 411-421. Le traité a longtemps été attribué à Gerson et inclus dans certains de ses manuscrits (Lille, Bibliothèque municipale, ms. 539, par exemple).

${ }^{21}$ «Elle agit avec habileté, comme si elle était un chef expérimenté, exercé à la méthode de l'art militaire». 
ser sous silence la bravoure au combat de la Pucelle, de peur d'en parler mal ou faussement ». Cela ne l'empêche pas un peu plus loin d'évoquer le combat d'Orléans où Jeanne fut associée à une petite armée qui sembla au bout de trois jours seulement ne former qu'un tout, jeune, vigoureuse et pleine d'allant ${ }^{22}$. Il ne va pas jusqu'à dire qu'elle en était le chef. C'est le bras de Dieu qui a agi. Il était difficile de ne pas reconnaître en 1429 le rôle primordial joué par Jeanne d'Arc au sein de l'armée de Charles, néanmoins c'était une constatation pratiquement impossible à exposer sans fard.

Les traités postérieurs à la condamnation de Rouen ont pour seule fonction de démembrer pièce à pièce les arguments de l'accusation en prouvant leur entière fausseté. L'article LIII de la première série de chefs d'accusation ne se retrouvait pas tel quel dans les douze assertions finales qui furent soumises à l'opinion de l'Université de Paris et au verdict des juges à Rouen, puis récusées par l'annulation du jugement. La présomption de se présenter comme chef de guerre ne fut donc pas reprise comme telle et pour elle-même par les théologiens consultés. Cependant l'article II de l'accusation, au détour du rappel de l'épisode du «signe» révélé à Charles VII, notait bien que cette révélation détermina le prince à croire aux assertions de Jeanne et à accueillir la Pucelle «pour mener les combats " ${ }^{23}$, mais il passe sans appuyer. Les traités, rédigés après que les armées royales menées par de très classiques nobles militaires de carrière eurent combattu jusqu'à la reprise de la Normandie et la victoire de Castillon, n'avaient plus à défendre le choix de la place de Jeanne au sein des troupes, mais bien plutôt à faire accepter sa seule présence en vêtements d'homme et coiffure ronde. Les auteurs, théologiens et juristes, ont donc généralement passé la question de Jeanne, chef de guerre, sous silence. Cela ne veut pas dire qu'ils n'abordent pas l'accusation de cruauté dirigée contre Jeanne, ni celle d'avoir porté les armes, mais leur argumentation dévie en général immédiatement vers les habits d'homme et la confusion des $\operatorname{sexes}^{24}$.

Discrets furent Théodore de Lelier, Jean Lhermite, Paulus Pontanus, Gui de Verceil (tempore quo Puella militiae se accincit ${ }^{25}$ ) et Guillaume Bouillé, dont les traités sont d'ailleurs fort courts ${ }^{26}$. Plus intéressant est le silence de Thomas Basin. $\mathrm{Au}$ long d'un discours, plutôt prolixe par ailleurs ${ }^{27}$, il refuse visiblement de s'occuper des éléments matériels de l'histoire de Jeanne d'Arc, qu'il a rejetés en fin de la seconde partie de son développement. Les aspects militaires sont encore réduits aux vêtements, portés à la demande de Dieu, destinés à protéger non seulement

${ }^{22}$ Revera humanitatus impossibile reputabatur, visa exercitu pompa, robore pugnatorum, dictorum fortitudine armatorum, concordia animorum, specie juvenum, residuum orbis quod ipsa triduo egit in uno mense agere posse (Ce qu'on disait humainement impossible à obtenir en un mois, grâce à l'ordre dans l'armée, la puissance des combattants, la résistance des armes et la concorde des âmes, spécialement des jeunes, elle l'obtint en trois jours).

${ }^{23}$ Procès, éd. Champion, t. II, p. 272 : ad eam recipiendum, pro bellis agendis.

${ }^{24} \mathrm{~V}$. R. Hotchkiss, Clothes make the Man, Female cross Dressing in Medieval Europe, New York, 1996, p. 49-68.

${ }^{25}$ Mémoires ..., p. 86 : «à l'époque où la Pucelle se préparait à l'état militaire».

${ }^{26}$ Summarium domini Theodorici, dans Mémoires..., p. 17-23 ; Opinio domini Johannis Heremite, ibid., p. 73-81 ; Opinio magistri Guillelmi Bouillé, ibid., p. 323-349.

${ }^{27}$ Opinio et consilium Thomo Basini, ibid., p. 187-235. 
la vertu de la Pucelle, mais également à éviter la concupiscence aux hommes qui l'entouraient. L'obéissance à Dieu, la préservation contre les tentations et les péchés occupent tout l'argumentaire de l'évêque de Lisieux.

Jean Brehal semble vouloir consacrer un paragraphe tout entier à la carrière militaire de la Pucelle (quod habitum virilem gestavit et bellis se immiscuit ${ }^{28}$ ), mais sur la trentaine de lignes qu'il emploie, trois seulement portent sur la guerre. Elles reprennent les paroles de Jeanne qui se saisissait de son étendard afin de ne tuer personne.

Élie de Bourdeilles ${ }^{29}$ fait preuve de quelque originalité en argumentant non pas sur la cruauté ou la présomption, mais en relevant et récusant l'accusation de sédition $^{30}$. Ici sont mis en avant pour justifier l'aventure de la Pucelle les arguments de la guerre juste et de la révolte nécessaire contre la tyrannie, thèmes souvent et douloureusement discutés au long des guerres civiles entre Armagnacs et Bourguignons $^{31}$. C'est à ce propos que passe sous la plume de l'évêque de Périgueux la défense d'une Jeanne que l'on aurait à tort accusée d'avoir causé des troubles, attenté à la paix et organisé le tumulte en menant le plus souvent l'armée au combat (ducendo saepius exercitus ad bellum). Troubles, tumultes et guerre étaient pourtant justifiés par le devoir de se dresser contre un régime tyrannique. Dans ces conditions la faute de sédition tombe d'elle-même, le rôle dirigeant de la Pucelle dans l'action ne mérite plus qu'on s'y arrête, ce qui fut sans doute un soulagement pour le théologien.

Martin Berruyer, en revanche, dans un discours très construit et pédagogique, aborde à plusieurs reprises le rôle militaire de Jeanne d' $\mathrm{Arc}^{32}$. En affirmant dans son premier chapitre que Jeanne dans tout ce qu'elle faisait était mue par un esprit supérieur et non humain, il pouvait effectivement insister non seulement sur son étonnante habileté aux armes (qui montrait que l'Esprit Saint était sur elle jusque dans ces petites choses), mais aussi sur l'exactitude de ses prédictions concernant ses propres actions quod ipsa levaret obsidionem coram Aurelianis positam ${ }^{33}$. L'accomplissement des prédictions des voix concernant la mission militaire de Jeanne a manifesté également l'origine divine des messages et des messagers. Le royaume était dans un état pitoyable: «villes désertes, maisons vides, campagnes incultes, églises sans desservants ni culte. Ni paix, ni sécurité, partout la terreur, dedans l'épouvante, dehors le glaive; non seulement à découvert mais au sein des cités mêmes se déroulaient de très cruels carnages de gens et quantité de bestiales effusions de sang de Chrétiens, tandis que la vertu gisait, foulée aux pieds par les impies $»^{34}$. En obéissant aux voix envoyées par Dieu, Jeanne rendait totalement licite son initiative belliqueuse : «[elle] suscitait une juste querelle, juste était ce prétexte de guerre ». Le succès attestait du caractère surnaturel et bénéfique de l'intervention

\footnotetext{
${ }^{28}$ Summarium fratris Johannis Brehalli, ibid., p. 97 : « de ce qu'elle portait un vêtement masculin et se mêlait au combat».

${ }^{29}$ Consideratio Helioe de Bourdeilles, ibid., p. 172-173.

${ }^{30}$ Procès, éd. Champion, t. 1, p. 355, Accusation, § VI.

${ }^{31} \mathrm{~B}$. Guenée, Un meurtre, une société. L'assassinat du duc d'Orléans, 23 novembre 1407, Paris, 1992 (coll. Bibliothèque des histoires), p. 189 et suiv.

${ }^{32}$ Opinio Martini Berruier, dans Mémoires..., p. 237-268.

${ }^{33}$ Ibid., p. 241 : « [elle disait] qu'elle lèverait elle-même le siège mis devant Orléans ».

${ }^{34}$ Ibid., p. 246.
} 
de la Pucelle. Les villes tombaient d'elles-mêmes, «de lions, les soldats devenaient des agneaux, on voyait se lever une aube nouvelle de paix et de sécurité ». La rhétorique permet à Berryuer de n'aborder ni les lieux ni les temps précis, de se complaire en d'élégants balancements de périodes et, comme ses collègues, de défiler des chapelets de citations bibliques et canoniques. Sans crainte de se contredire en soulignant d'abord les remarquables aptitudes au combat de l'héroïne, il finit par accabler la jeune fille sous une pluie de lénifiantes épithètes : aimant l'unité, prônant la paix, haïssant l'effusion de sang, pieuse, douce, clémente, soumise ${ }^{35}$. L'évêque du Mans ne fait que remplir ici le rôle d'un avocat de la défense qui choisit ses arguments et abandonne les aspects de l'action exercée par la Pucelle qui ne conviennent pas aux circonstances du moment.

Les mêmes réticences et occultations se lisent chez Robert Ciboule et Jean Bréhal, toujours en écho aux accusations de sédition et de cruauté. Les deux opposent à l'argument du port et de l'usage des armes offensives, de la fréquentation des gens d'armes, voleurs, assassins et sacrilèges, celui de la nécessité des armes défensives et offensives contre l'oppression, comme le montrent les exemples antiques et bibliques $^{36}$.

Déborah, car il faut bien finir par elle, demeure pour le sujet qui nous occupe la référence préférée de nos auteurs. Ce n'est que par artifice qu'à l'imitation des astuces de présentation de ces théologiens nous abordons en dernière partie son histoire en la comparant à celle de Jeanne. Le Livre des Juges rapporte que Dieu abandonna Israël, en raison de ses fautes, à l'oppression des Cananéens. Cela dura vingt ans, vingt ans de sujétion à une armée «aux neuf cents chars bardés de fer $»^{37}$. La prophétesse et juge Déborah fit venir vers elle Baraq et lui dit que Yahvé ordonnait de rassembler des hommes : la victoire leur serait assurée sur Sisera, le chef de l'année cananéenne. Baraq refusa de partir sans que Déborah l'accompagnât, elle le suivit donc. Au jour annoncé par Déborah, Baraq et ses dix mille hommes écrasèrent les ennemis. Sisera s'enfuit et «toute l'armée de Sisera tomba sous le tranchant de l'épée et pas un homme n'échappa». Le cantique de Deborah et de Baraq célèbre la victoire et l'exploit de Yahel, épouse d'Heber, qui tua Sisera en fuite ${ }^{38}$. On note tout de suite un certain nombre de coïncidences avec l'histoire du $\mathrm{XV}^{\mathrm{e}}$ siècle : les armées de Baraq sont dites riches de dix mille hommes comme celles que le roi aurait confiées à Jeanne (selon ses dires à elle). Déborah est une prophétesse avérée, une inspirée de Dieu comme le reconnaissent les partisans de Charles VII pour Jeanne. Comme celles de Jeanne, ses prédictions se sont révélées exactes. Comme la $\mathrm{Pu}$ celle, elle a accompagné les armées jusqu'au lieu des combats. Là s'arrête le parallélisme des destins, là surgit pour les théologiens la difficulté de faire entrer Jeanne dans le moule si confortable des références scripturaires. Déborah n'est pas une combattante, encore moins un chef de guerre. Elle ne conduit pas l'armée, elle

\footnotetext{
${ }^{35}$ Ibid., p. 255.

${ }^{36}$ Consideratio Roberti Ciboule, ibid., p. 374-375.

${ }^{37}$ Juges, $1,23$.

${ }^{38}$ Sur la comparaison entre Jeanne d'Arc et Yahel, voir F. Michaud-Fréjaville, «L'effusion de sang dans les procès et les traités concernant Jeanne d'Arc », Le sang au Moyen Age, Cahiers du C.R.I.S.I.M.A., $\mathrm{n}^{\circ} 4,1999$, p. 332-340, réimpr. supra, article $\mathrm{n}^{\circ} 15$.
} 
l'accompagne, elle est sans armes. Elle n'a eu qu'une seule initiative, fondamentale certes : la transmission de la parole de Yahvé annonçant la victoire prochaine, puis fixant le jour de la bataille. Pour le reste, c'est Baraq qui lui demande de l'accompagner, qui poursuit un ennemi mis en déroute par la crainte de Dieu.

À un moment ou un autre de leur développement, et ce dès 1429, les théologiens qui réfléchirent sur le personnage de Jeanne utilisèrent l'exemple de Déborah. En revanche les juristes n'ont pas privilégié ce modèle parmi leurs références et on ne rencontre pas le cas de Déborah chez Théodore Lelier, Paul Pontanus, Jean Lhermite, Jean Bouchard, Jean de Montigny ou Guillaume Bouillé. Les autres présentent de façon assez diverse un épisode biblique qu'il s'agissait de donner comme antécédent plausible à l'épopée de Jeanne d'Arc.

Gerson est d'une prudence extrême: Déborah est une des preuves que l'événement qui se déroule dans l'été de 1429 n'est pas entièrement d'ordre inouï puisque des épisodes similaires ont pris place dans l'Histoire: «ainsi, dans tous ces exemples prend communément place quelque chose de naturel $\aleph^{39}$. Mais Gerson ne pouvait ignorer que Déborah était une prêtresse réputée que depuis longtemps le peuple venait consulter, qu'elle n'avait pas surgi de l'inconnu, qu'elle avait convoqué Baraq et que celui-ci s'était rendu à cette injonction. Gerson était trop fin pour insister plus longtemps.

Martin Berruyer, Jean Brehal et Élie de Bourdeilles, plutôt que l'évocation ambiguë de Déborah marchant avec le général Baraq et les troupes d'Israël, préfèrent citer les mêmes versets ou fragments du cantique de Baraq et Deborah : "Les âmes courageuses avaient lâché prise, elles s'étaient assoupies, alors se leva Déborah, elle se leva la mère en Israël, le Seigneur choisit de nouveaux combats et Il abattit les portes des ennemis $\gg^{40}$ (Juges, 5, 7-8). On doit avouer que la référence maternelle convenait fort mal à Jeanne pour laquelle on mettrait plutôt en avant une certaine fragilité de la jeunesse ${ }^{41}$. En revanche, l'évocation du réveil de la vaillance d'un peuple à l'appel d'une prophétesse convenait parfaitement à la situation du royaume de Bourges quand survint la Pucelle.

Le rapprochement entre les carrières militaires des deux femmes grâce au rappel d'une présence de Déborah au sein des combats est à peine effleuré par Thomas Basin: Déborah, au côté du fils d'Abinoam, a combattu contre Sisera (incit proelium $)^{42}$. Guy de Verceil use d'une formule plus compliquée, véritable paraphrase du style biblique, qui semble supposer une présence physique de la prophétesse sur le champ de bataille lui-même: «Dieu remit dans la main de Déborah l'armée de Jabin, roi de Canaan, et à elle on attribua la victoire au nom des fils d'Israël $»^{43}$. Cette présence paraissait néanmoins douteuse à Jean Dupuy. Si l'image de Déborah s'était tout de suite imposée à lui, dès les débuts de l'histoire incroyable de la Pucelle et les

\footnotetext{
${ }^{39}$ Exempta possent de Debora [...] in quibus ut communiter miscetur semper aliquid naturale. ${ }^{40}$ Traduction littérale sur la Vulgate de Cessaverant fortes in Israhel et quieverunt / donec surgeret Debbora surgeret mater in Israhel / Nova bella elegit Dominus et portas hostes ipse subvertit. Cf. Martin Berruyer, Mémoires..., p. 251 ; Jean Brehal, ibid., p. 449.

${ }^{41} \mathrm{~F}$. Michaud-Fréjaville, «Johanna puella, La "jeunesse" de Jeanne d'Arc dans les traités en sa faveur », Études médiévales, n ${ }^{\circ}$, Amiens, 2000, p. 327-332, réimpr. supra, article ${ }^{\circ} 14$.

${ }^{42}$ Mémoires..., p. 219.

${ }^{43}$ Ibid., p. 86.
} 
victoires inespérées sur les Anglais, ces Cananéens, il ne pouvait cependant se prêter à l'inflexion d'un texte sacré pour le rendre adéquat aux nécessités du temps présent. Il se sentit donc obligé de remarquer que nulle part on ne lisait que Déborah avait littéralement prêté main-forte à Baraq ${ }^{44}$.

C'est exactement à la suggestion inverse que tente d'arriver Robert Ciboule dans le plus long développement que ces traités aient consacré à Jeanne guerrière. Le souci de Ciboule est de montrer que la jeune fille n'a pas agi contre Nature. Agir contre nature serait - en tous cas - illicite, or la légalité de l'action de Déborah n'est l'objet d'aucune remise en question. Dans l'Ancien Testament, on lit que beaucoup de femmes auraient pris les armes et conduit des guerres (bella duxisse). En utilisant un vocabulaire de l'action - Déborah s'est soulevée (surrexit), elle a marché jusqu'au bout (perrexit) avec Baraq et a soumis (expugnavit) Sisera -, Ciboule insiste sur le rôle viril d'une femme qu'il prend soin de ne pas rendre exceptionnelle. Pour renforcer la concordance de l'Ancien Testament avec le temps de Jeanne, il ne craint pas d'affirmer que, selon une autorité incontestable, Alexandre de Halès, et une référence vérifiable, le livre II de sa Summa theologiae, Déborah aurait «usé de vêtements masculins et des armes de combat pour repousser les ennemis du peuple d'Israël $»^{45}$. La place d'une Jeanne chef de guerre, dux exercitus, de l'armée de Charles VII est donc pleinement légitimée. Robert Ciboule est bien le seul en son temps à le dire avec une telle assurance.

Les réticences des autorités consultées pour le procès en réhabilitation rejoignaient presque les indignations des juges de Rouen. Il est certain que la transgression des rôles, assumée avec tant d'audace par la Pucelle, était à leur yeux difficilement justifiable, d'autant qu'il n'y avait pas de véritables antécédents, ni dans le monde légendaire dont on savait bien l'irréalité, ni dans l'Ancien Testament, sauf à gauchir la lettre de celui-ci, ce qui était une pente dangereuse. Le procès en annulation de la condamnation de Jeanne avait bâti son argumentation non pas sur la reconnaissance d'une femme forte, morte pour avoir sauvé un royaume chrétien, mais sur la démonstration de l'iniquité du supplice infligé à une innocente écrasée par un tribunal injuste. Pour les savants, mieux valait passer avec souplesse, éluder et laisser retomber le silence sur les aspects militaires de la Pucelle, les témoins de sa vie se chargeraient bien de transmettre l'émotion et la passion populaires, qu'avait déjà comprises Christine de Pizan, devant la simple jeune fille si experte à la guerre qu'ils avaient vue en tête des troupes royales.

${ }^{44}$ Non tamen legitur quod ipsa Deborah aliquid manualiter egerit.

${ }^{45}$ Ibid., p. 375 : Quod Deborah utebatur veste virili et armis militaribus ad expugnandos inimicos... La citation de Juges, 6, 7-8, Cessaverunt fortes in Israel, vient ensuite. 